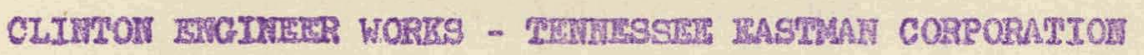

Procowa Iruprovement Division

Technteal Report

TITLE : CLEAIIIG E-WITTS BY BLASFIEG

ABSYRAOT : An alx-wator-abresive blasting symten thoroughly cleans copper parts of If unita vithout damage to the parts. Ittile abrestve is reguired because of the continvous recyole of the abracive sluxry.

AUTHORS :

Jack R. MeWharter, $\mathbf{x} / 5$

Stophen Yorezunis, $\mathrm{g} / 5$

Txperiment pexzormed Decenber 14 to Decembor 20, 1944

Reported to Dr. J. P. Bexter on Januazy 13, 1945

Report Ro-1.oued: Soptember 8, 2945

This report was prepared as a scientific account of Government-sponsored work. Neither the United States, nor the Commission, nor any person acting on behalf of the Commission makes any warranty or representation, express or implied, with makes ant the use of any inforact mation, apparatus, method, or process disclosed in this report may not infringe privatelyowned rights. The Commission assumes no liability with respect to the use of, or from damages resulting from the use of, any information, apparatus, method, or process disclosed in this report.
Photostat Price $\$ 3.30$

Microfilm Price $\$ 2,40$

Available from the

Office of Technical Services

Department of Commerce

Washington 25, D. C. 


\section{DISCLAIMER}

This report was prepared as an account of work sponsored by an agency of the United States Government. Neither the United States Government nor any agency Thereof, nor any of their employees, makes any warranty, express or implied, or assumes any legal liability or responsibility for the accuracy, completeness, or usefulness of any information, apparatus, product, or process disclosed, or represents that its use would not infringe privately owned rights. Reference herein to any specific commercial product, process, or service by trade name, trademark, manufacturer, or otherwise does not necessarily constitute or imply its endorsement, recommendation, or favoring by the United States Government or any agency thereof. The views and opinions of authors expressed herein do not necessarily state or reflect those of the United States Government or any agency thereof. 


\section{DISCLAIMER}

Portions of this document may be illegible in electronic image products. Images are produced from the best available original document. 


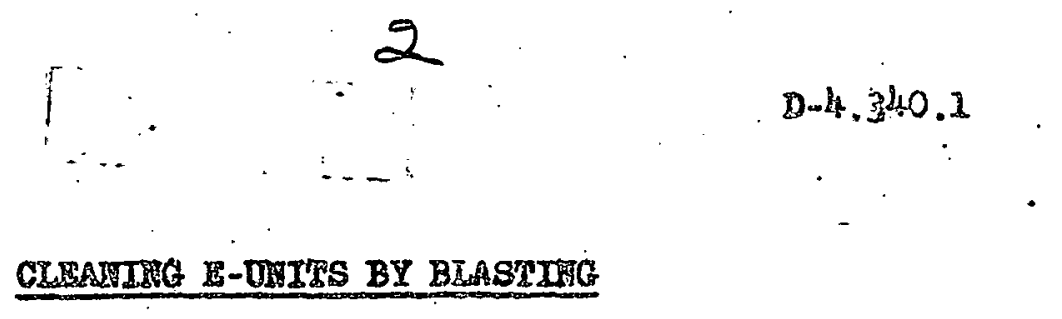

Dofect : So remove B-

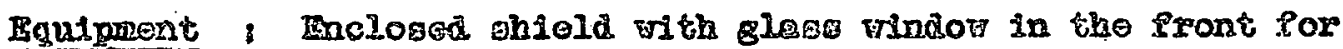

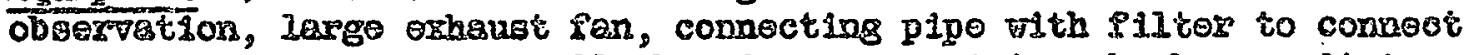

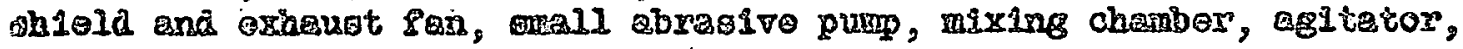
nozalo.

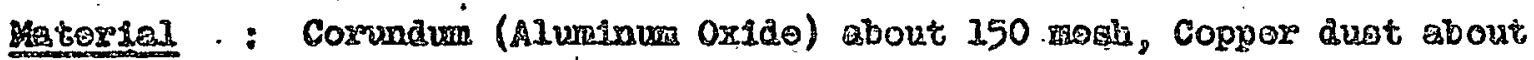

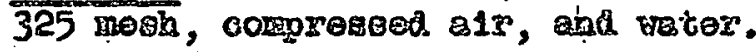

Procedure : The aleaning unit consigtod of a lango ancioaed shleld vith e

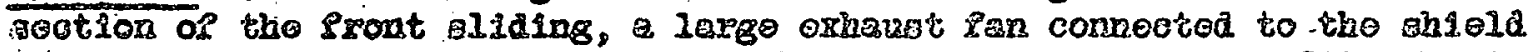

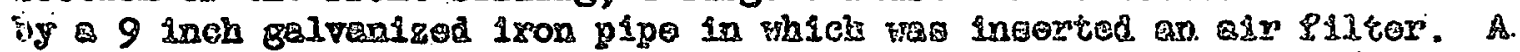

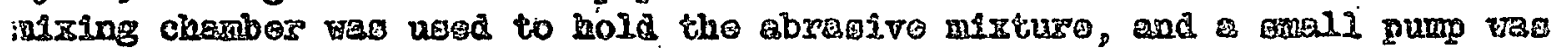

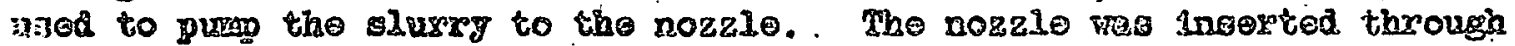
the gllaing portion af the front of tho ablold and realed to this elide by

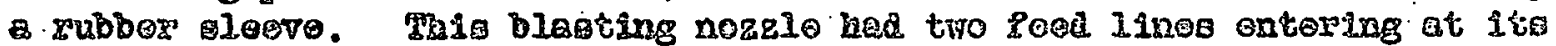
inanisold. Through one lino a 50 by volgkt mixture of corrandum and veter -

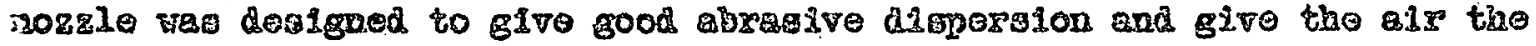

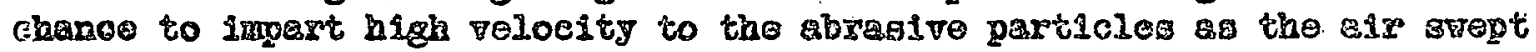
hy the ebreite outlot. The high volooity partieles or abreasto leaving

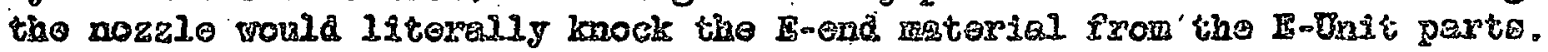

The composed of entering the ehteld ver pulted frou the whiold through

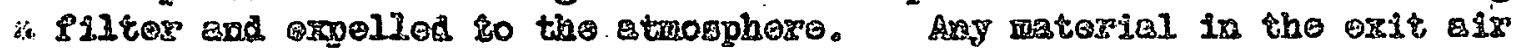
whould be removed by the Pilter to the ashast 1 ine.

The wet obragive ester gitriking the target foll to tho bottor of tho ohiold

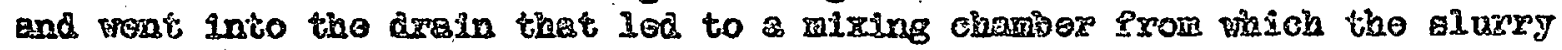

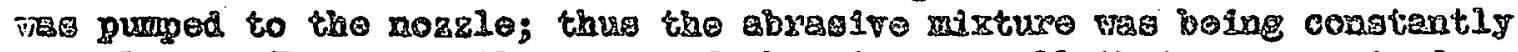

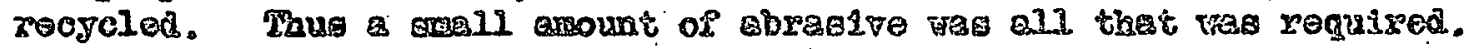

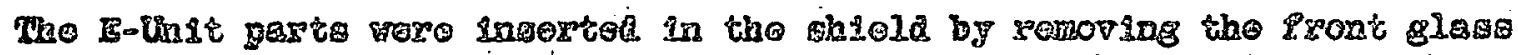

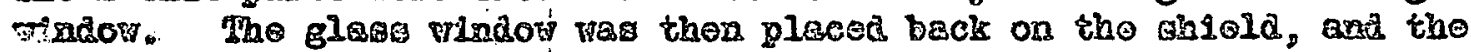

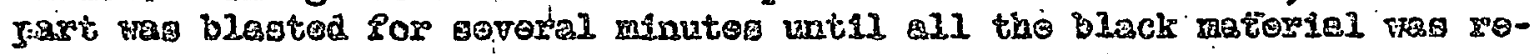

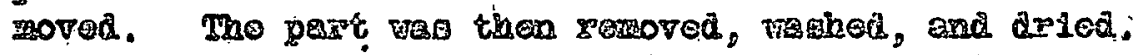

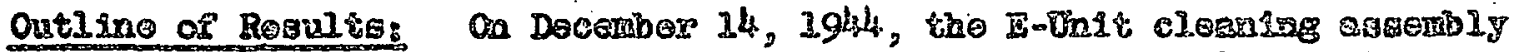

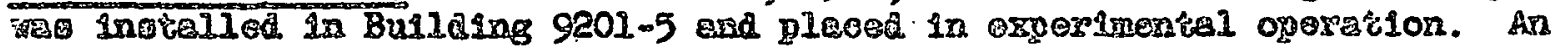

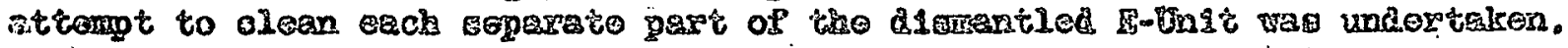

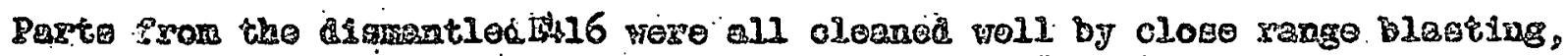
the nozzle to tazgot diotane varying sxou $2^{n}$ to $5^{\mathrm{n}}$. Who outlot of the

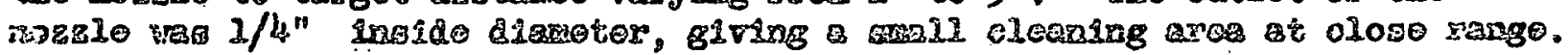

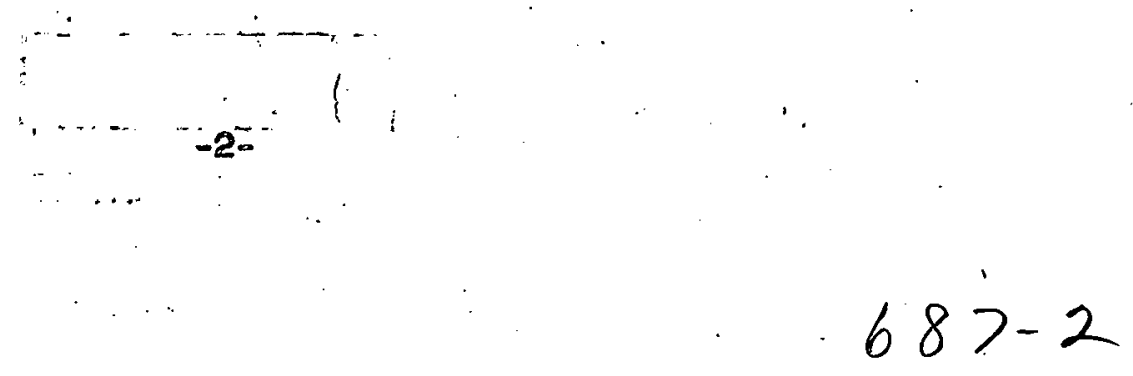




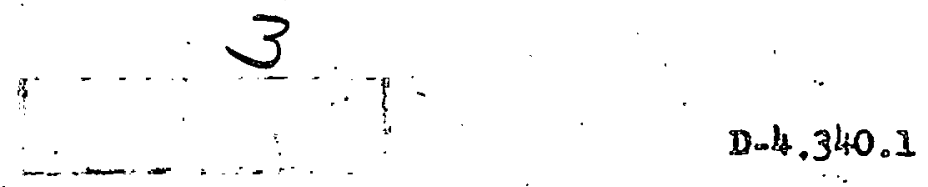

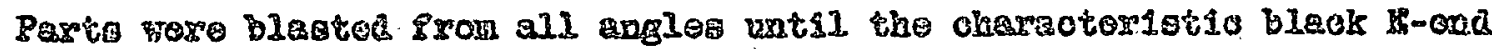

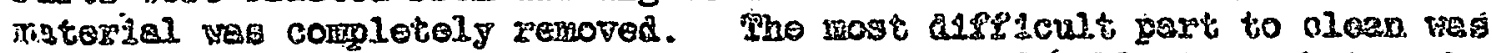

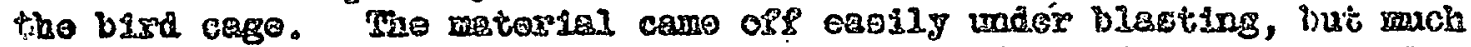

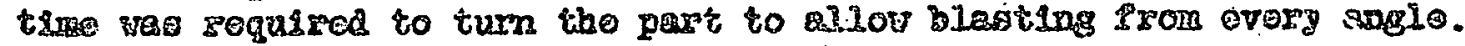

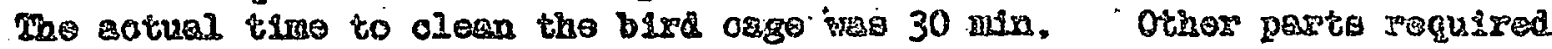
1.DE tirio.

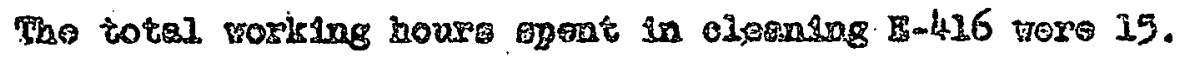

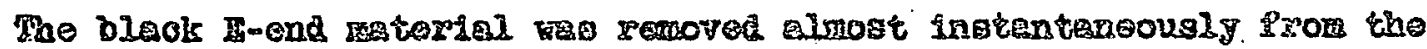

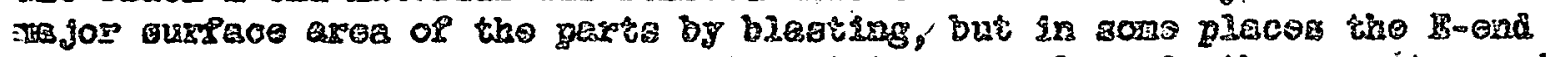

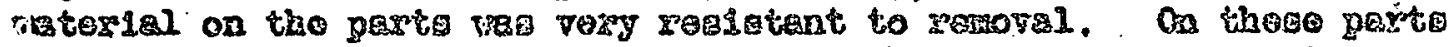

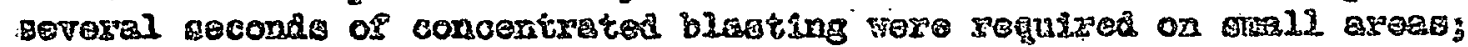

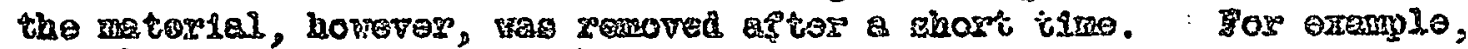
one pitoh on a birs cago cover hat mataral cared on lt tinet wes hard to

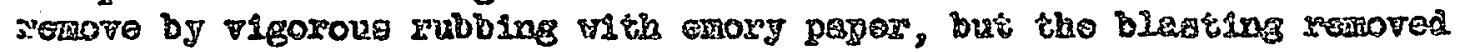
thie patorial aptor sovaral ninutos.

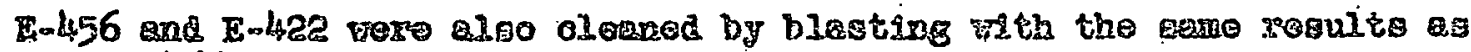

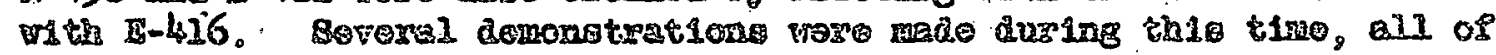
sinduh ohored tho desired zosulte.

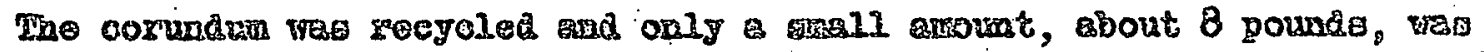

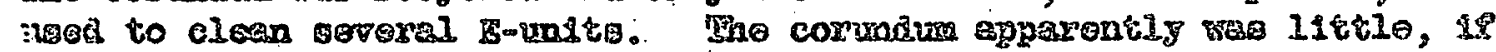
day, afseoted by tho blasting. Itw oloaning property wes retained oven after 20 os 25 kours of ueo.

A guxdy wa

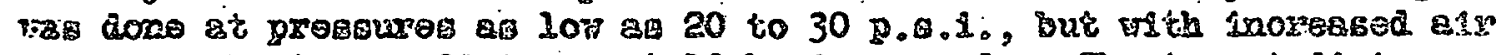

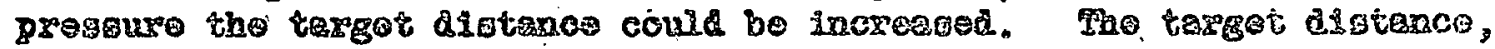

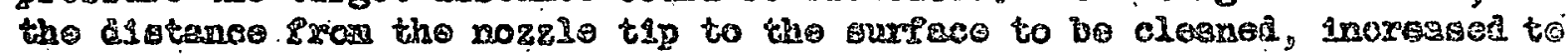
i.

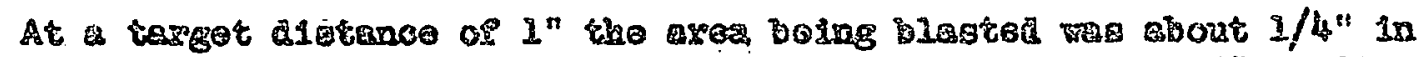

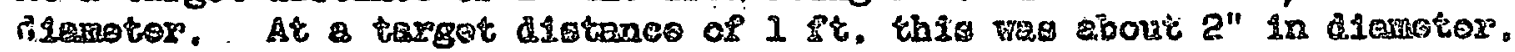

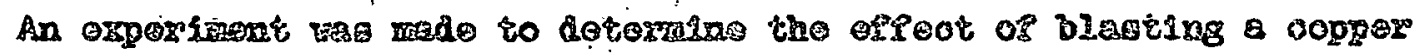

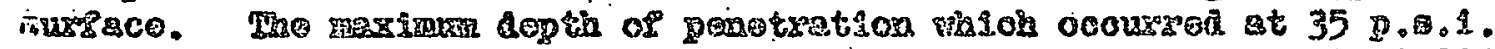

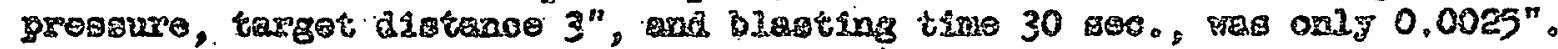

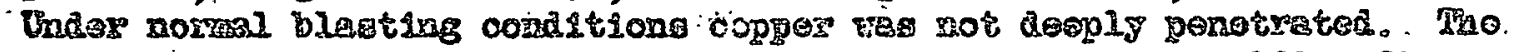

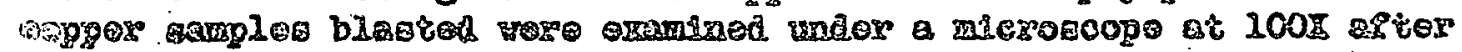

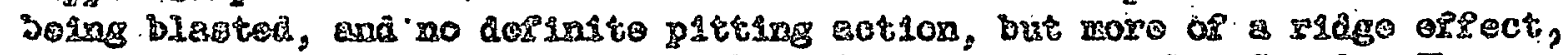

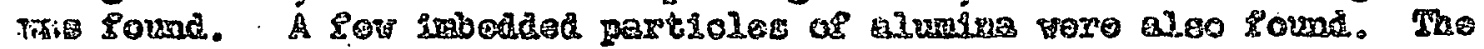

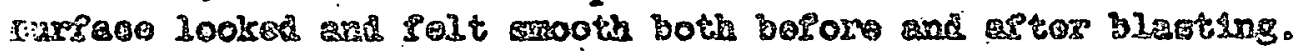

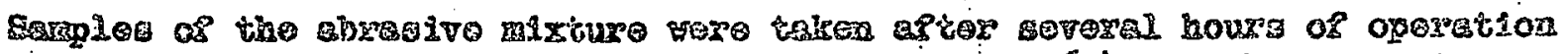

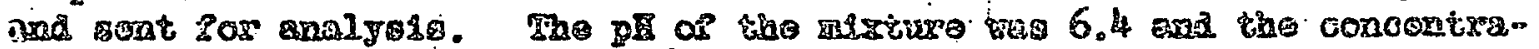

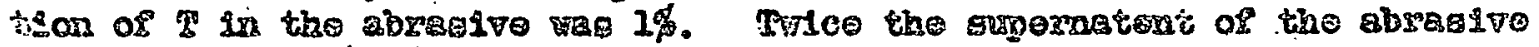

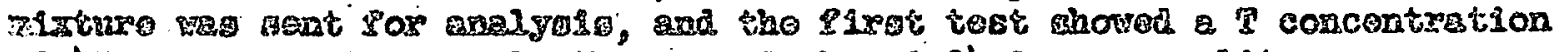

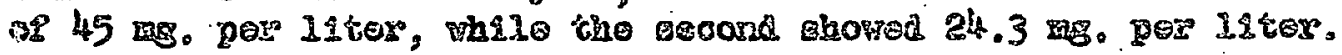




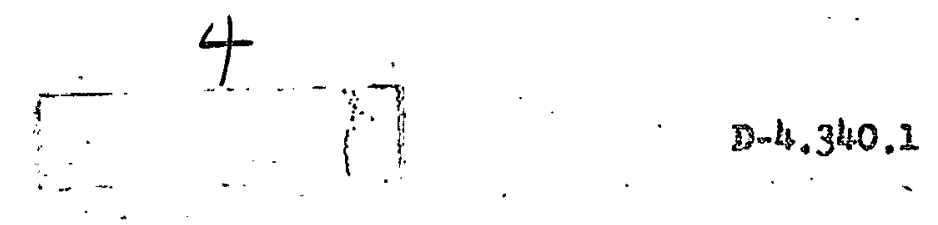

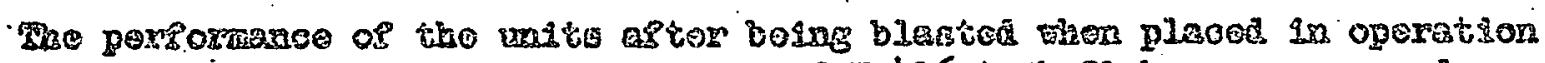

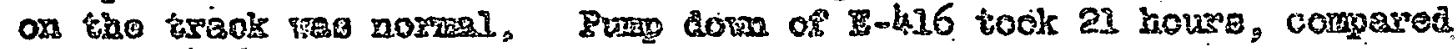
vith ats.6 kour trak evorage.

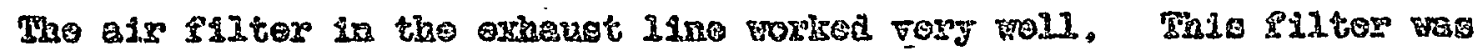

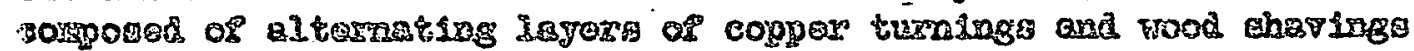

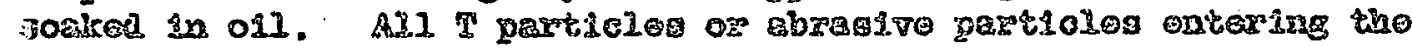

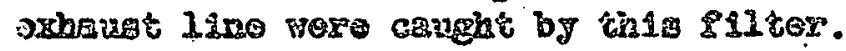

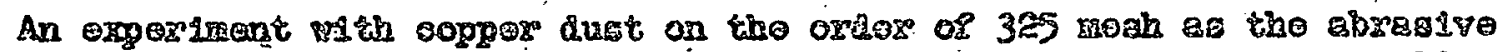

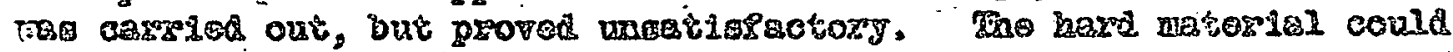
tie zanove onit by giolonged blagting.

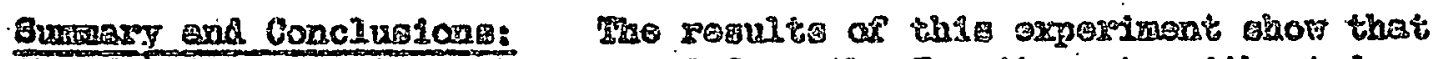

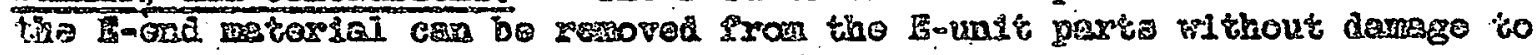
the pasto.

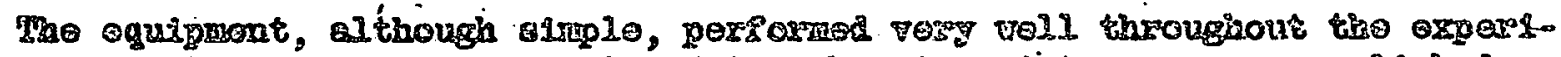

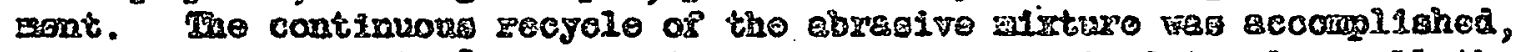

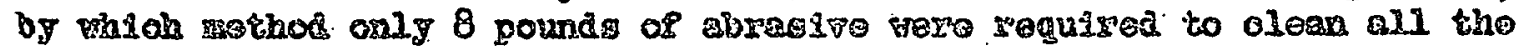

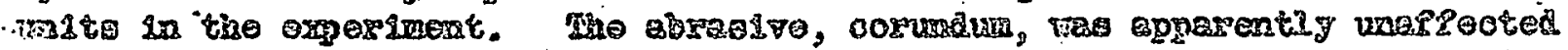

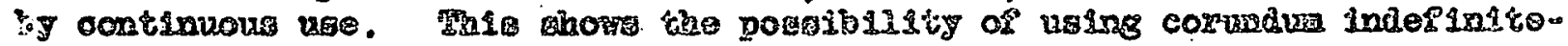

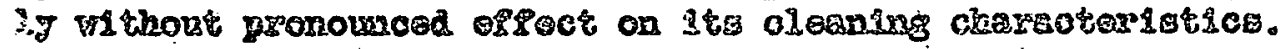

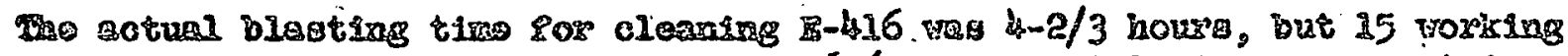

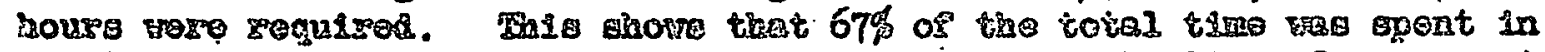

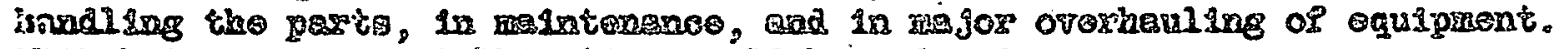

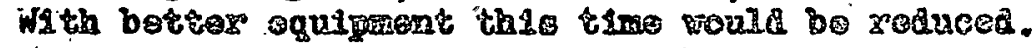

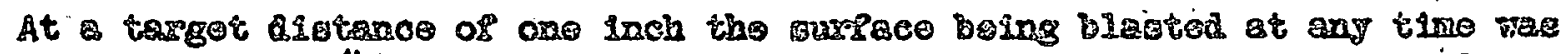

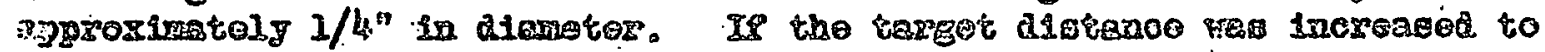

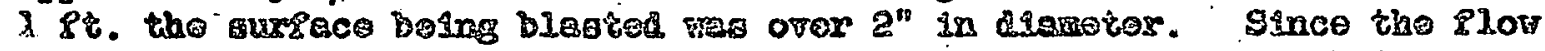

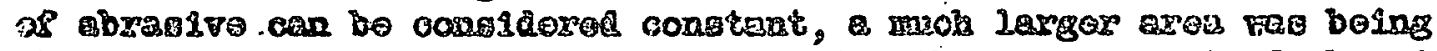

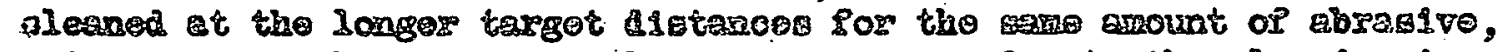

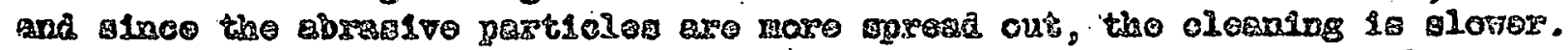

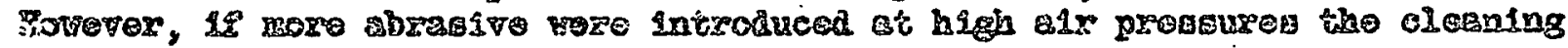

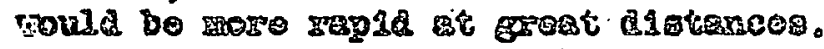

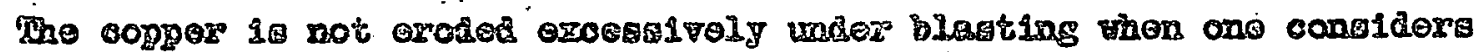

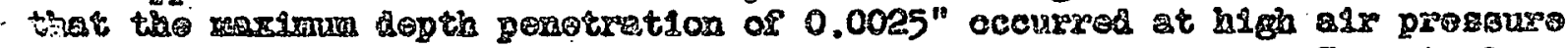

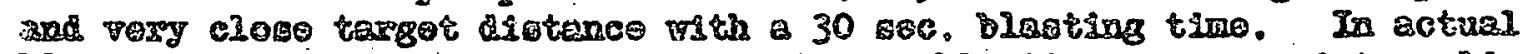
blesting the longeat porsos of comcentrated blastiag on any point roula

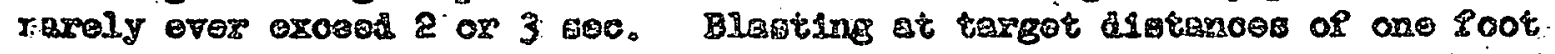

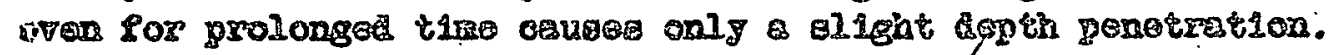

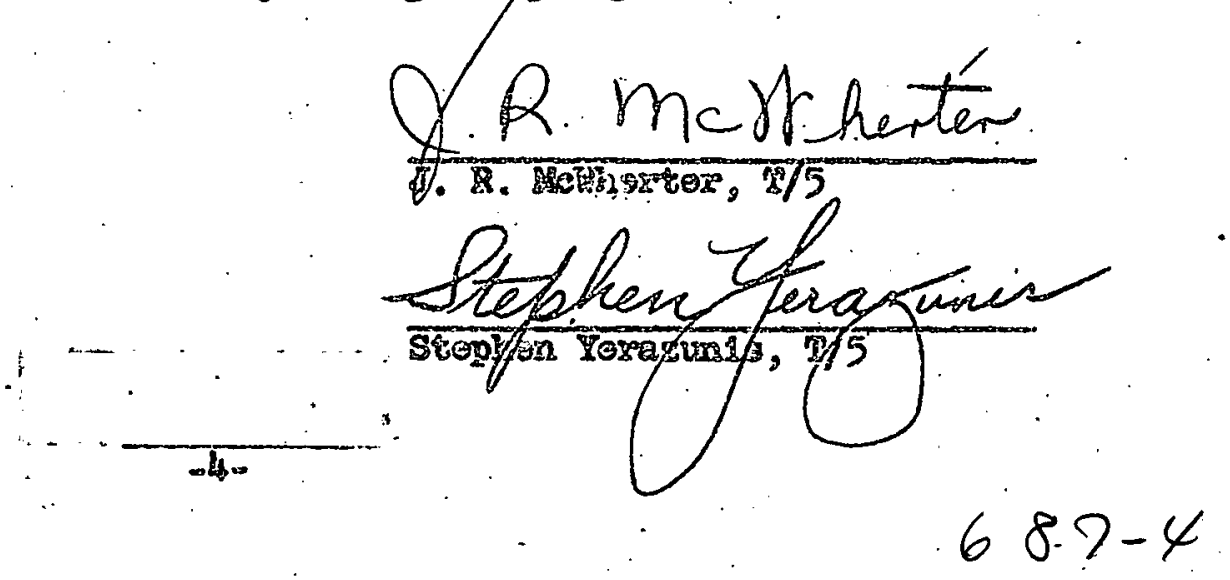




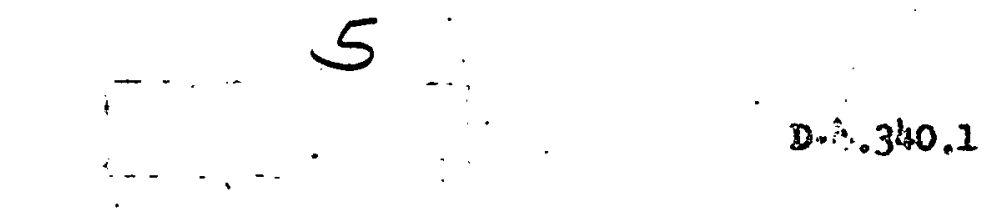

\title{
OBTOHFAT DATA
}

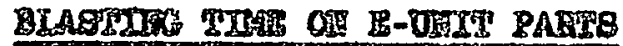

\author{
EAPE \\ B1rd Cage \\ Sereon Exa Bals \\ Coppor Ccolas Por 8-Can \\ 81de shutter Bolder \\ grainlegs stoel Part
}

APs: $10: 1$
30 min.
15 min.
10 mina.
5 min.
5 min.

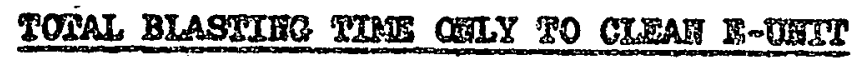

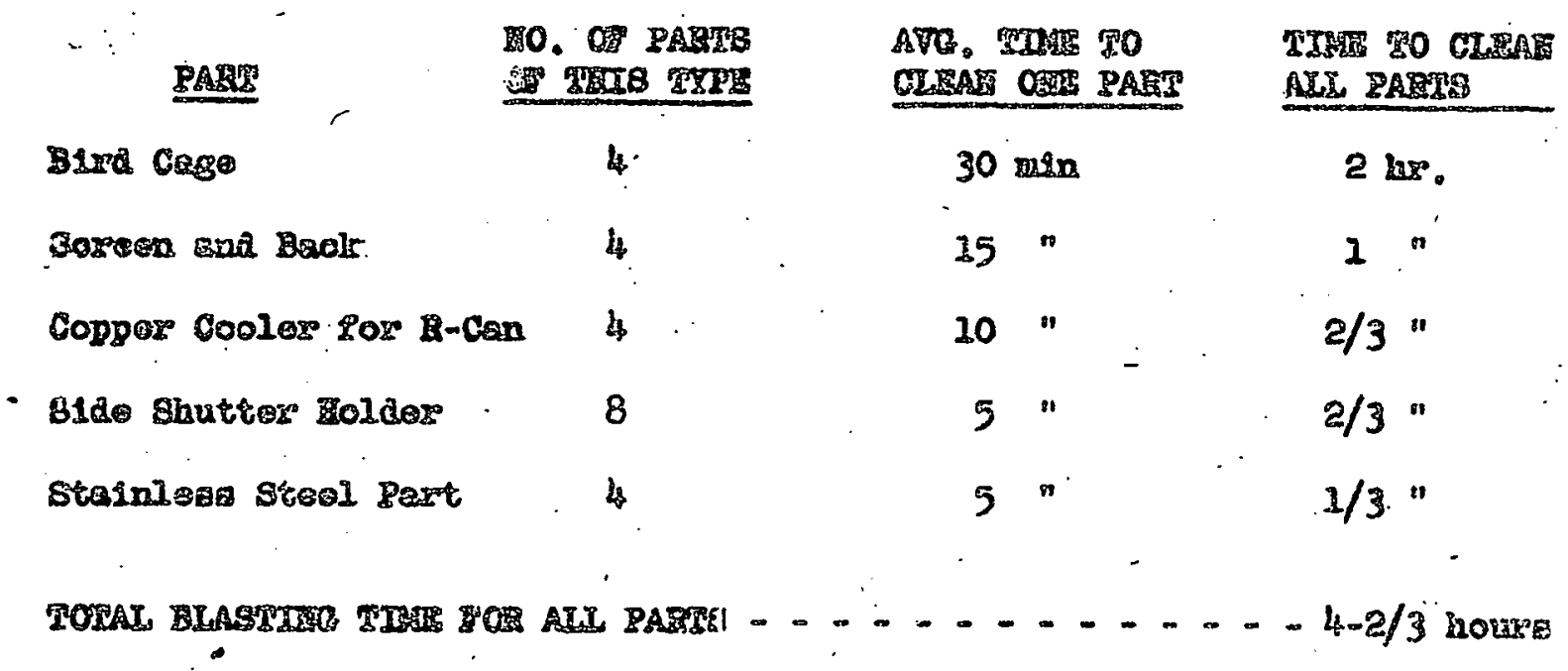




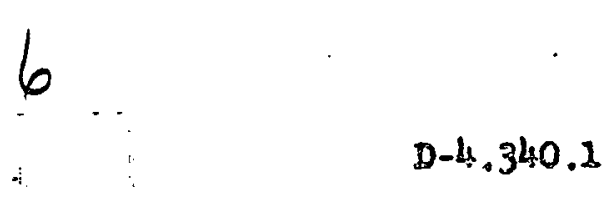

ORIOIFAT DATA

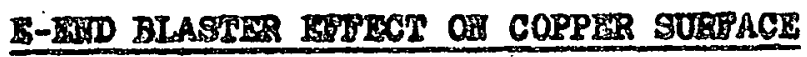

\begin{tabular}{|c|c|c|c|c|c|c|c|c|}
\hline & $\begin{array}{l}\text { Barmiolo } \\
\text { So. }\end{array}$ & $\begin{array}{l}\text { Targot } \\
\text { Diat.in. }\end{array}$ & $\begin{array}{l}\text { Prese. } \\
\text { p.0.1. }\end{array}$ & $\begin{array}{l}\text { Tine } \\
\text { dee. }\end{array}$ & $\begin{array}{l}\text { Ores. th. } \\
\text { Grame. }\end{array}$ & $\begin{array}{c}\text { Pinel Wt: } \\
\text { Grane }\end{array}$ & $\begin{array}{l}\text { Asea } \\
\text { in }\end{array}$ & $\begin{array}{l}\text { Max. Dopth } \\
\text { of Ponotratios } \\
\text { in. xalo-3 }\end{array}$ \\
\hline & 1 & 3 & 25 & 10 & 14.1759 & 14.1623 & .601 & .5 \\
\hline & 2 & 3 & 25 & 20 & 14.0175 & 13.9837 & .601 & 1.25 \\
\hline & 3 & 3 & 25 & 30 & 14.0054 & 13.9384 & .994 & 2.0 \\
\hline & 14 & 3 & 35 & 10 & 14.0370 & 14.0096 & .785 & 1.0 \\
\hline & 5 & 3 & 35 & 20 & 13.6060 & 13.5214 & .785 & 1.5 \\
\hline . & 5 & 3 & 35. & $30^{\prime}$ & 13.3542 & 13.2407 & .994 & 2.5 \\
\hline & 7. & 12 & 35 & 60 & 13.5292 & 13.6705 & 2.750 & 1.0 \\
\hline & 3 & 3 & 25 & 10 & 14.1623 & 14.1365 & .785 & .5 \\
\hline$\dot{0}$ & 9 & 3 & 25. & 20 & 13.9837 & 13.9451 & .785 & .75 \\
\hline$\dot{3}$ & 10 & 3 & 25 & 30 & 13.9384 & 13.8935 & .994 & 1.0 \\
\hline & .21 & 3 & 35 & 10 & 14.0096 & 13.9816 & .785 & 2.0 \\
\hline . & 3.2 & 3 & 35 & 30 & 13.2407 & 13.1736 & 1.230 & .5 \\
\hline & 23 & 12 & 35 & 60 & 23.4705 & 13.4010 & $2.750^{\circ}$ & 1.0 \\
\hline
\end{tabular}

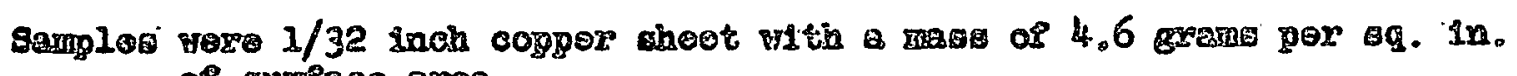
of muxpece arra. 


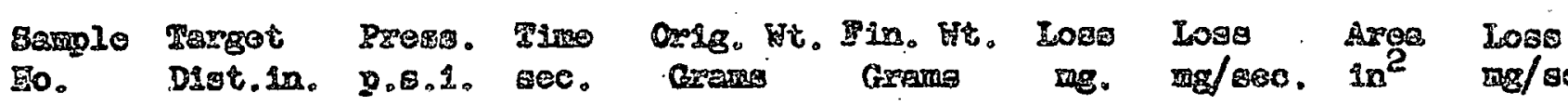

D.

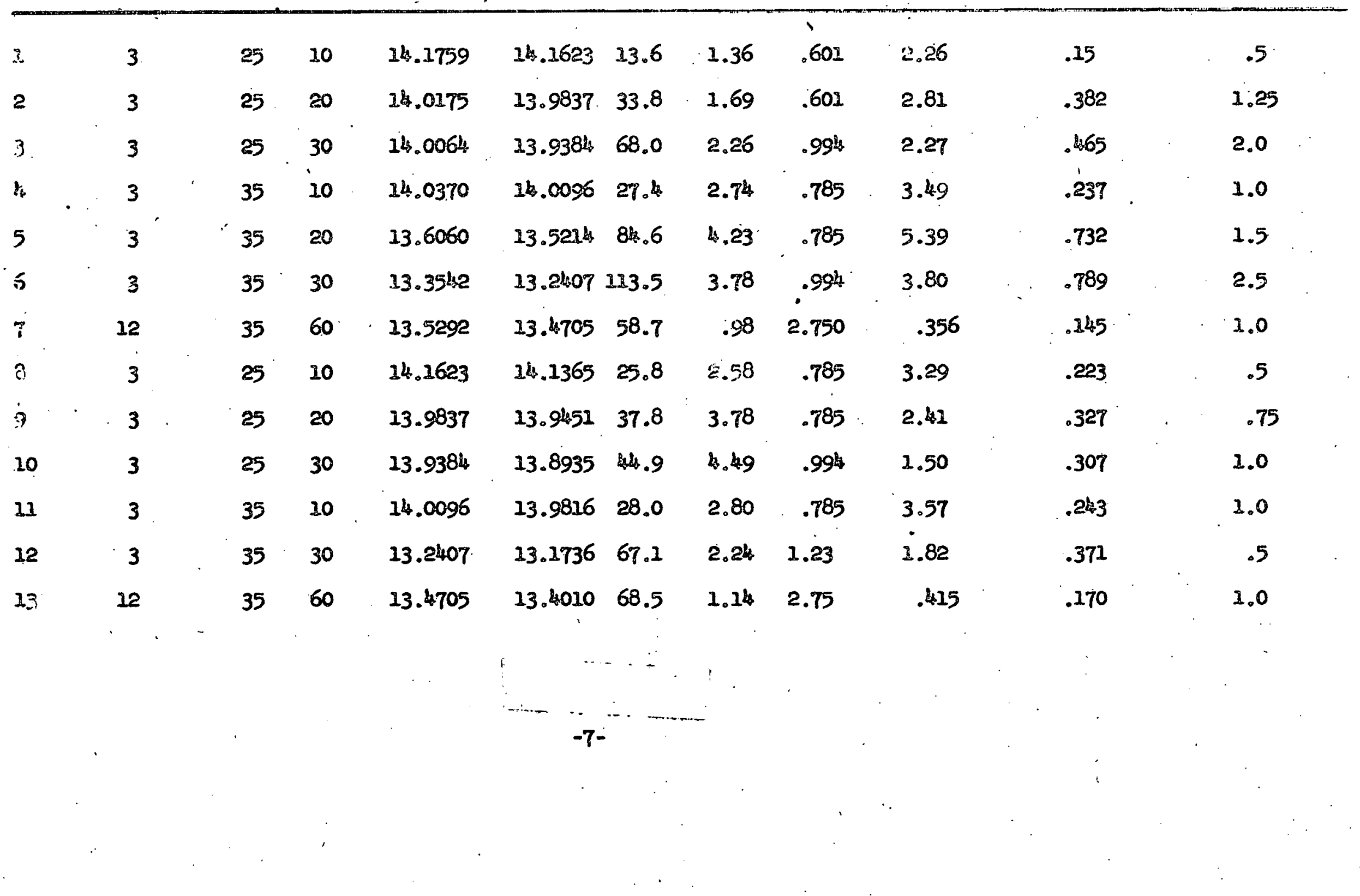




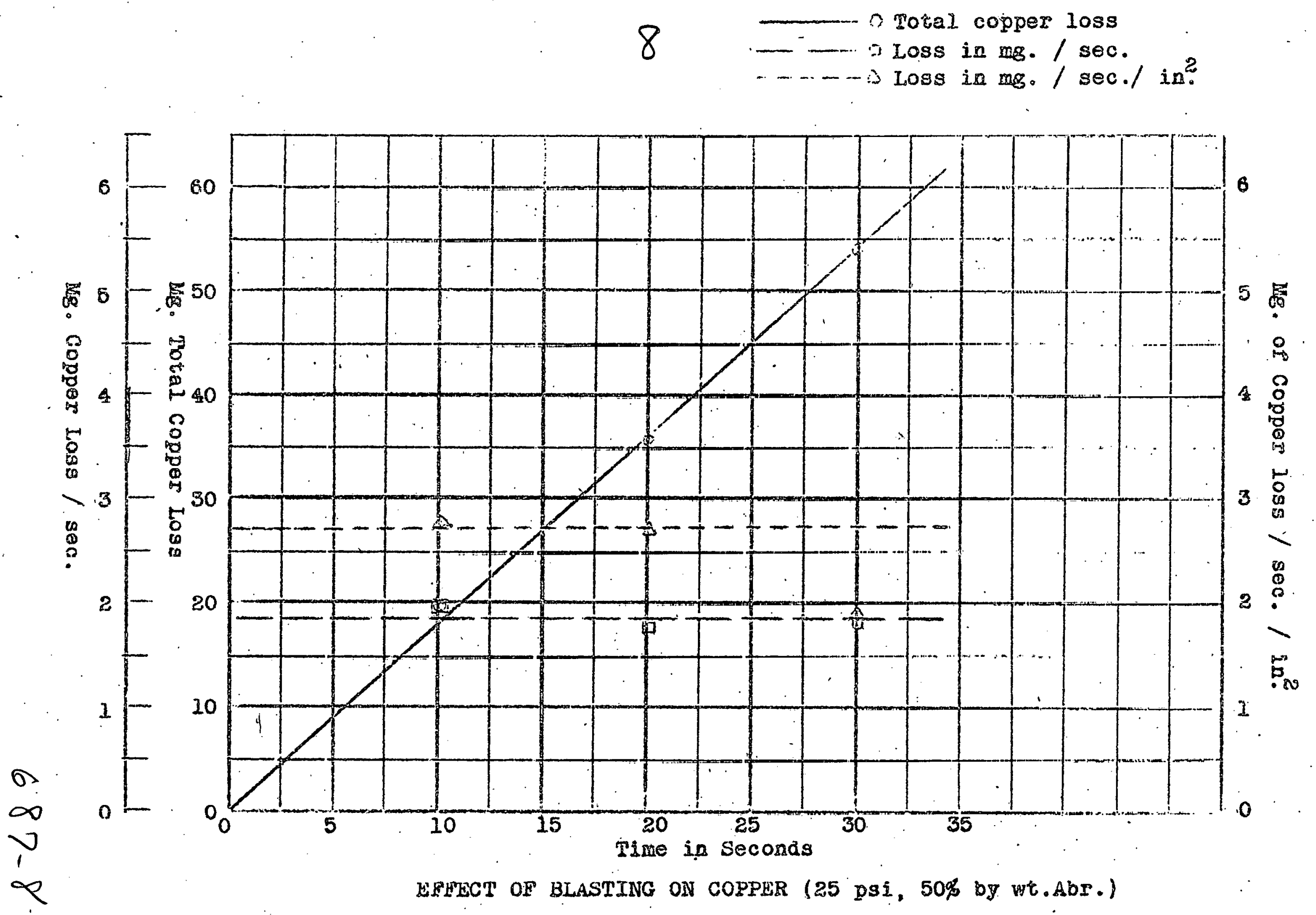



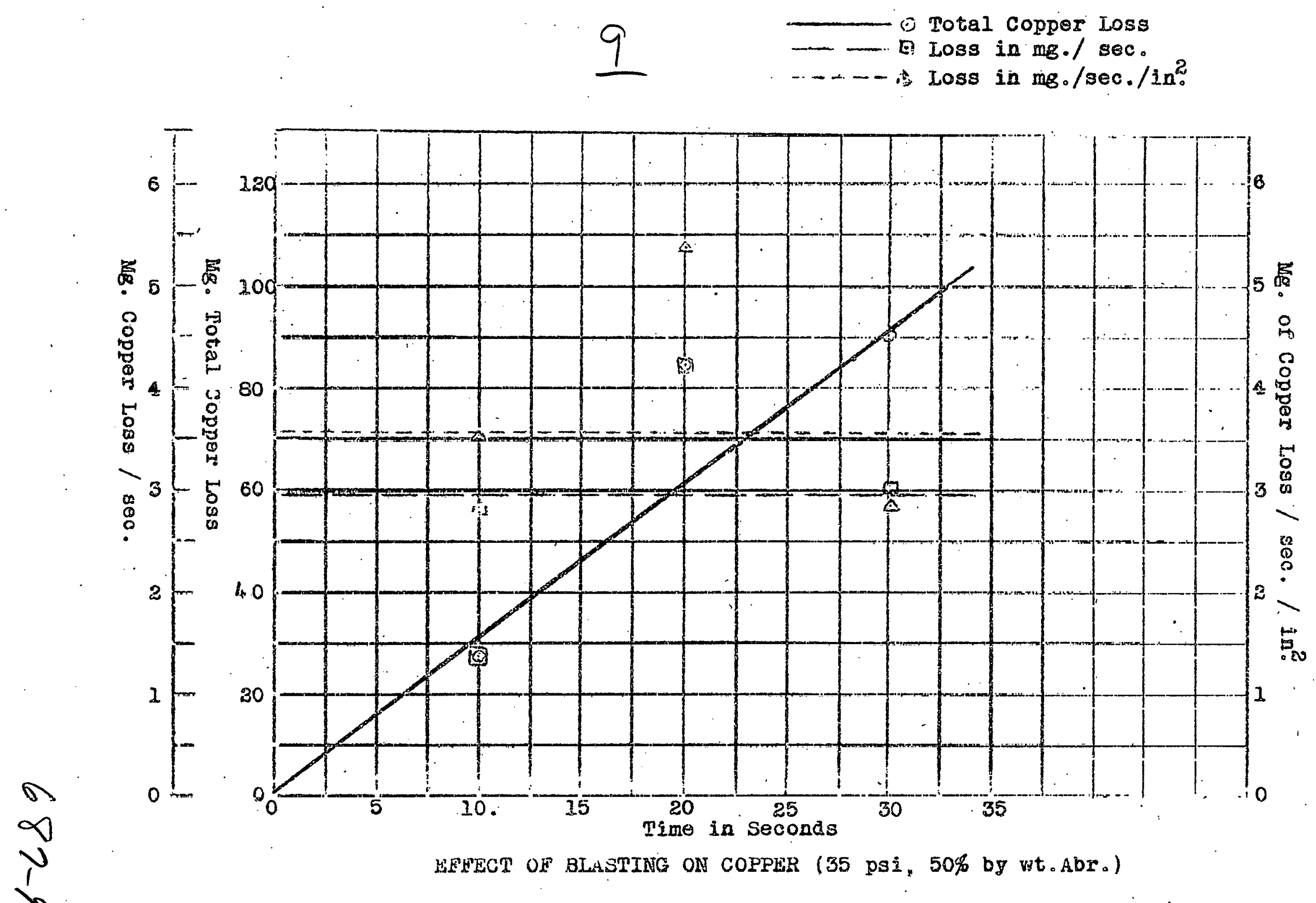


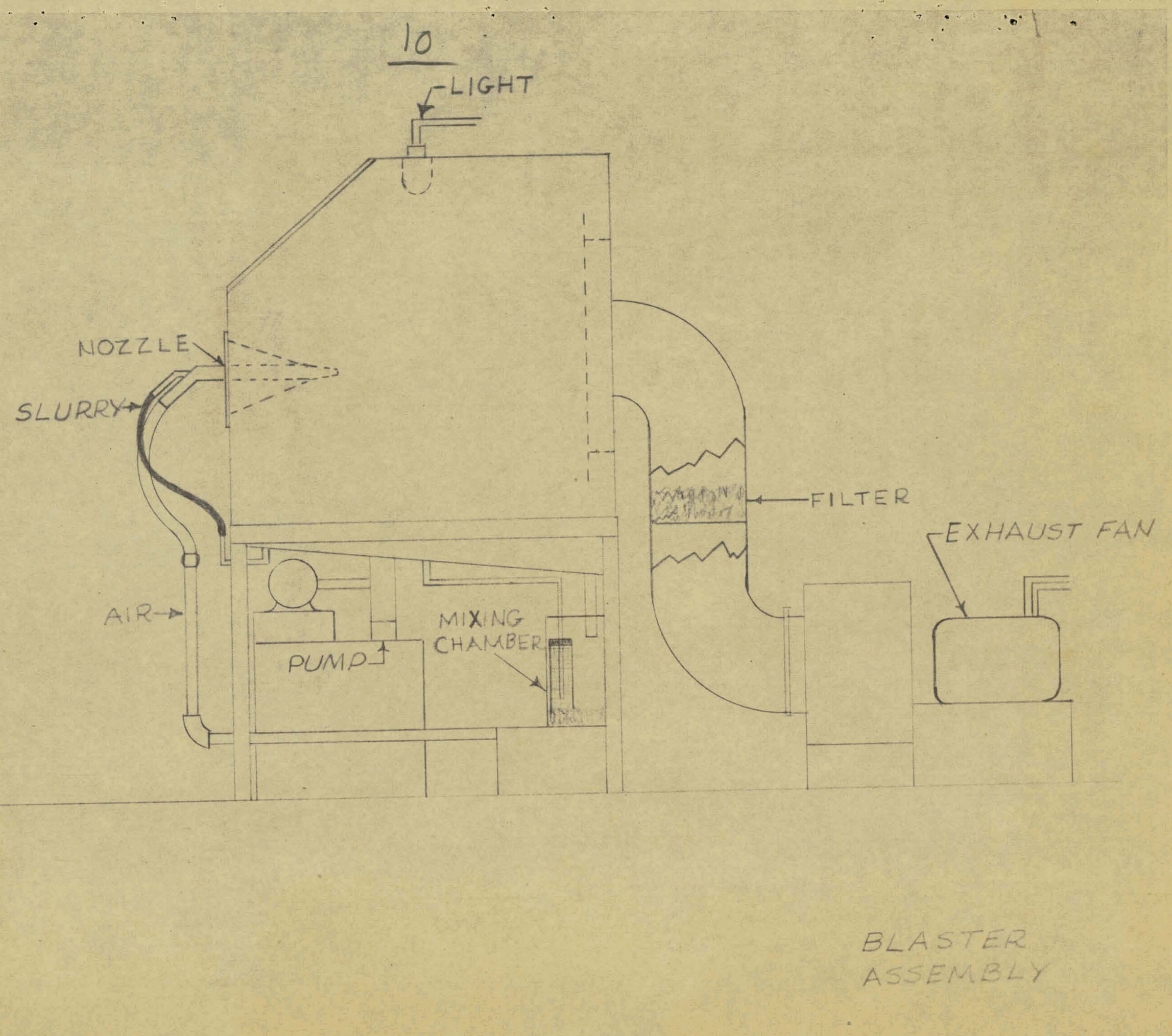


THIS PAGE

\section{WAS INTENTIONALLY}

LEFT BLANK 